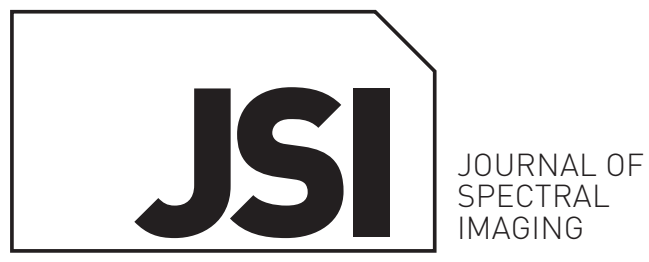

Paper Presented at IASIM 2016, July 2016, Chamonix, France

\title{
openaccess
}

\section{Hyperspectral photoluminescence imaging of defects in solar cells}

\author{
Ingunn Burud, ${ }^{\mathrm{a},{ }^{*}}$ Torbjørn Mehl, ${ }^{\mathrm{a}}$ Andreas Flo, ${ }^{\mathrm{a}}$ Dominik Lausch ${ }^{\mathrm{b}}$ and Espen Olsen ${ }^{\mathrm{a}}$ \\ aNorwegian University of Life Sciences, Department of Mathematical Sciences and Technology, Campus Ås, Pb 5003, 1433 Ås, Norway. \\ E-mail:ingunn.burud@nmbu.no \\ ${ }^{b}$ Fraunhofer-Center für Silizium Photovoltaik CSP Otto-Eißfeldt-Straße 12, 06120 Halle (Saale), Germany
}

\begin{abstract}
The present work is a demonstration of how near infrared (NIR) hyperspectral photoluminescence imaging can be used to detect defects in silicon wafers and solar cells. Chemometric analysis techniques such as multivariate curve resolution (MCR) and partial least squares discriminant analysis (PLS-DA) allow various types of defects to be classified and cascades of radiative defects in the samples to be extracted. It is also demonstrated how utilising a macro lens yields a spatial resolution of $30 \mu \mathrm{m}$ on selected regions of the samples, revealing that some types of defect signals originate in grain boundaries of the silicon crystal, whereas other signals show up as singular spots. Combined with independent investigation techniques, hyperspectral imaging is a promising tool for determining origins of defects in silicon samples for photovoltaic applications.
\end{abstract}

Keywords: near infrared, photovoltaic, multicrystalline silicon, MCR, PLS-DA

\section{Introduction}

Material defects in silicon wafers for photovoltaic (PV) applications can lead to significant reduction in the efficiency of the final solar cells due to a decrease in charge carrier lifetime. Uncovering these defects and the physical mechanisms behind them have high priority internationally in order to increase conversion efficiency. In-depth knowledge of material defects would allow crystallisation processes to be optimised and new methods to be developed to upgrade low quality wafers to yield solar cells with higher overall efficiencies.

Photoluminescence (PL) imaging without spectral resolution is a well-established, non-destructive technique for characterising multicrystalline silicon wafers and solar cells. ${ }^{1,2} \mathrm{~A}$ traditional PL image will display good areas with high lifetime of charge carriers with high photoluminescence intensity, and low lifetime (bad areas) with low intensity. The high intensity photoluminescence is a result of recombination between the valence band and conduction energy band for silicon, also called the band-to-band (BB) recombination. Spectral infor- mation has traditionally been obtained only from selected points on the wafer through near infrared (NIR) photoluminescence spectroscopy. Characteristic emission signals in these spectra are supposedly related to dislocations and are named D-lines. ${ }^{3}$ With hyperspectral photoluminescence imaging, both spatial and spectral distribution of the crystal imperfections across the whole wafer can be studied, as shown by Olsen \& $\mathrm{Flo}^{4}$ and Burud et al. ${ }^{5}$

In this work, we demonstrate how NIR hyperspectral photoluminescence imaging of silicon wafers and solar cells combined with multivariate image analysis techniques, such as multivariate curve resolution (MCR) and partial least squares discriminant analysis (PLS-DA), can be used to extract and to map a cascade of radiative defects in multicrystalline silicon samples. Moreover, we show how utilising a macro lens yields a spatial resolution of $30 \mu \mathrm{m}$ on selected regions of the samples, revealing detailed information on the defects. 


\section{Materials and method}

\section{Samples}

The samples named 1,2 and 3 in this article are multicrystalline Si solar cells, fabricated with a standard commercial process based on boron-doped $156 \times 156 \mathrm{~mm}^{2}$ wafers made from standard electronic grade silicon with comparable high purity.

\section{Hyperspectral photoluminescence imaging}

Photoluminescence imaging is based on the characteristic of a material, typically a semiconductor, that enables it to emit light in response to a light source with energy high enough to excite electrons from the valence band to the conduction band. Hyperspectral photoluminescence imaging of samples 1, 2 and 3 were obtained using a NIR hyperspectral pushbroom camera (SWIR, Specim, Finland) with a HgCdTe detector with spectral sensitivity in the $929-2531 \mathrm{~nm}(0.49-1.33 \mathrm{eV})$ range and a resolution of $6 \mathrm{~nm}$ (256 bands). As excitation source, an $808 \mathrm{~nm}$ line laser was used with an irradiated power density of $2 \mathrm{~W} \mathrm{~cm}^{-2}$ (Lasiris Magnum II). The laser has enough energy to excite the electrons from the valence band to the conduction band and the photoluminescence light that we measure results from all radiative recombinations of electrons to the valence band with energy larger than $0.49 \mathrm{eV}$. A $850 \mathrm{~nm}$ lowpass filter prevented laser beam reflections from entering the optic apparatus. The samples were cooled to $90 \mathrm{~K}$ on a polished aluminium surface on top of a cryogenic cooler filled with liquid nitrogen. The cooling quenches some of the nonradiative emissions and hence enhances the luminescent active imperfections. For most applications, the spatial resolution is around $500 \mu \mathrm{m}$. A macro lens was also used which yields an increased resolution to $30 \mu \mathrm{m}$. The hyperspectral imaging setup is shown schematically in Figure 1. This setup yields a three-dimensional data file Ispatial $x, y$ and spectral $\lambda$ ), called a hypercube.

\section{Electroluminescence imaging}

Electroluminescence imaging is similar to photoluminescence imaging except that the excitation source is an electrical current. Spectrally integrated electroluminescence images were acquired of sample 1. The images were obtained at room temperature using a camera with a charge coupled device (CCD) camera in order to detect band-to-band (with a Si detector) and sub-band defect luminescence lusing an InGaAs detector).

\section{Image analysis}

In order to extract all the different defect related emission signals from the hypercube, $\mathrm{MCR}^{6}$ was used. MCR is a mathematical method for deconvolving complex, convoluted signals composed of several discrete, simultaneously occurring signals. An iterative process optimises the best fit by an alternating least squares (ALS) algorithm. For each of the samples studied, an MCR analysis was carried out on the hypercube of

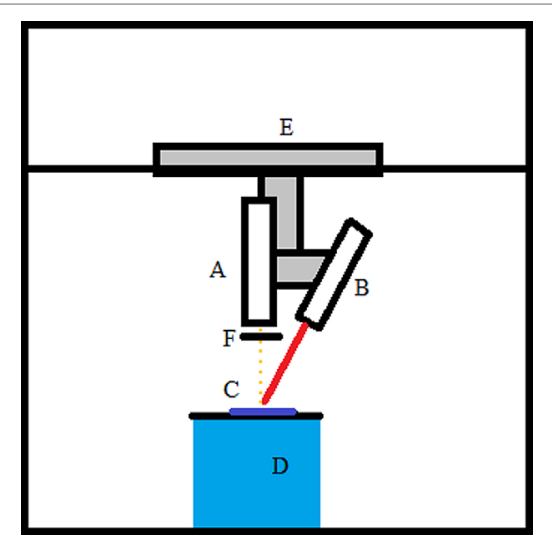

Figure 1. Schematic illustration of hyperspectral photoluminescence imaging. The figure shows the camera (A), the laser (B) illuminating the sample (C) placed on the cryogenic cooler (D). The translation stage (E) moves the camera and the filter (F) eliminates laser light to enter the optic apparatus.

256 photoluminescence images. In some cases, the goal is to identify a certain type of defect emission in samples. For this purpose, PLS-DA could also be applied. PLS-DA is a linear classification method, a derivative of the standard PLS regression that uses class variables instead of numeric variables. In this work, the PLS-DA algorithm in PLS Toolbox leigenvector. com) under the MATLAB environment was used. On the hypercube of sample 1, regions of Type $A$, Type $B$ and $B B$ signal [108 spaxels (pixel spectra)] and small spots of VID3 and D07 (9 spaxels) were manually selected, and the PLS-DA model was applied to all the other spaxels in the hypercube. The same PLS-DA model was then applied to an unknown solar cell, named sample 2. Unfortunately, we have no complementary measurements for validation of this sample.

\section{Results and discussion}

\section{Classification of defects}

The results from the MCR analysis of a solar cell (sample 1) are shown in Figure 2 together with an electroluminescence image of a neighbouring sample ${ }^{7,8}$ (coming from the same location in the silicon block from which they were cut). The loading plot in units of energy volts is shown in Figure 2a). The electroluminescence image (Figure $2 \mathrm{~b}$ ) can be compared with the score images for five components laid on top of each other, each with its own colour (Figure 2c). Two of the MCR components correspond to the two types of defects, named Type A and Type B that were found with the electroluminescence method. In addition, two other defects previously found in other samples and named D07 and VID3 were clearly detected, as well as the Si BB emission. In some pixels, there are slight overlaps between defect-related emission, and the most dominant is seen in the image in the figure. However, the defects classified as Type A and Type $B$ are very distinct in their location, and hardly any overlap of these emission signals are found. 


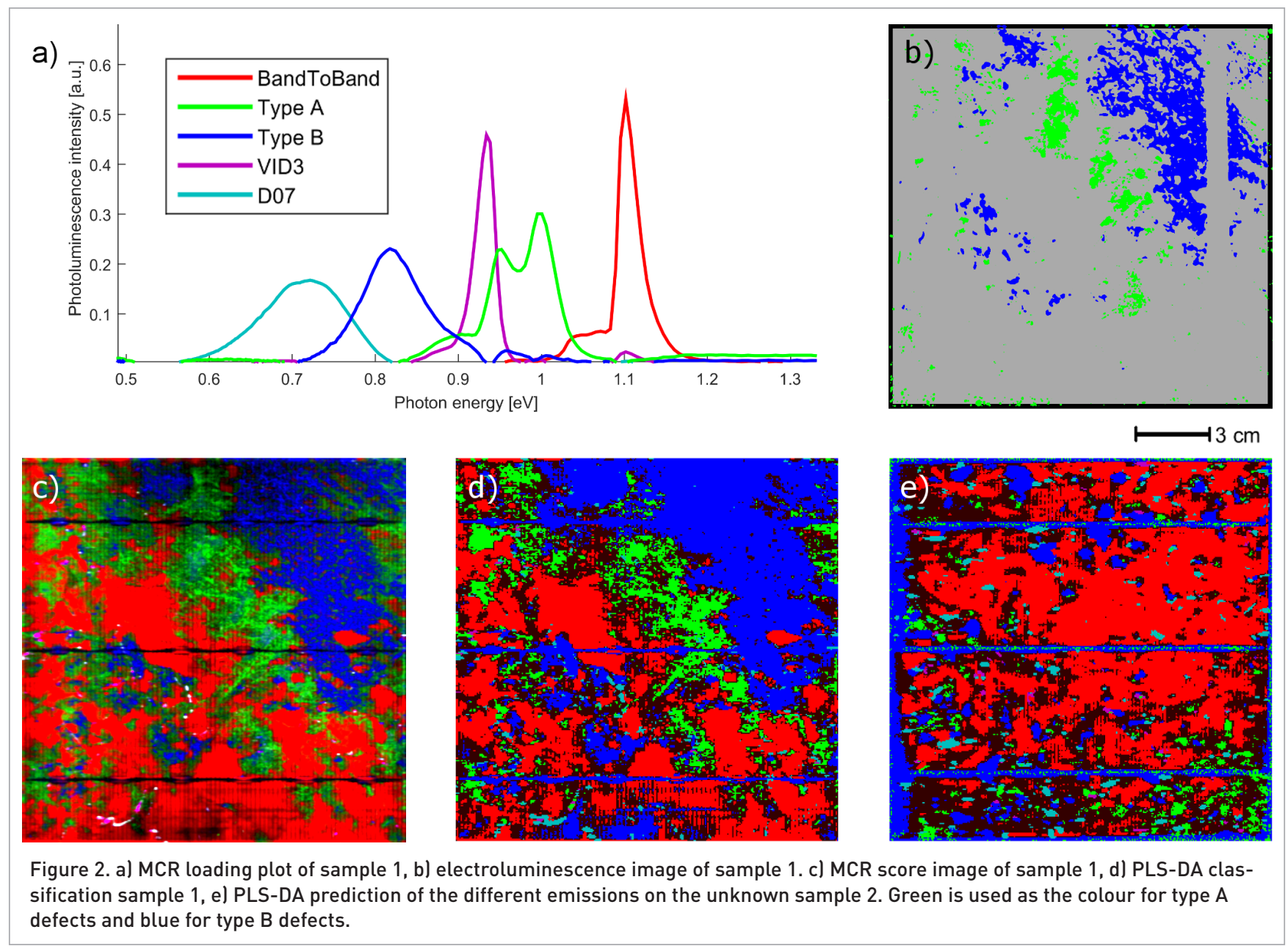

The same hyperspectral image was analysed with PLS-DA where regions of the four defects (type A, B, VID3 and D07) and BB were manually selected as classes. These selected regions were used to construct the PLS-DA model. The resulting classification is shown in Figure 2c and corresponds well with the results from the MCR analysis. The good agreement with the electroluminescence image in this example confirms that hyperspectral NIR photoluminescence imaging with MCR analysis is well-suited for application to silicon wafers and solar cells. Moreover, electroluminescence uses current to excite electrons and can only be carried out on finished solar cells with busbars mounted to conduct the electricity. Busbars are thin strips of silver that are screen printed onto the cells to collect and conduct the current generated by the solar cell. They can be seen as horizontal lines in Figure 2. Hyperspectral imaging, however, is not dependent on current since it uses the laser light to excite the electrons.

The PLS-DA model described above was also applied to another cell (sample 2) that has not previously been investigated by other methods. The five classes of luminescence emission could be identified also in sample 2. It can be seen that the busbars and the edges of the sample are misclassified as type B defect. This is due to the presence of second order harmonics of the laser light reflections in the busbars, at $0.77 \mathrm{eV}$, merging with the signal from type B defects. This indicates that the low-pass filter is not preventing all laser light entering the camera lens. Laser light $(808 \mathrm{~nm})$ will not be detected by the camera (929-2531 nm), but second order harmonics (1616 nm / $0.77 \mathrm{eV}$ ), generated in the optics, will. A new low-pass filter at $1000 \mathrm{~nm}$ has now improved this for future measurements.

\section{Cascade of energy levels}

According to the physics of recombination mechanism, one would expect defect-related emissions with many similar but slightly different energy levels, almost as a cascade of energy levels. It is therefore interesting that if one applies an MCR analysis with many components, a distribution of such energy levels is found, as shown in Figure 3 where the MCR loading plot and the score images for 11 components are shown for sample 3 . When looking at the score images, one sees that all the defect emission signals have different spatial distributions. These results indicate that the classification of defects such as type A, B and D-band lines are only on a macroscopic level. Investigations using independent techniques have to be carried out in order to reveal the possible different origins of these signals. 

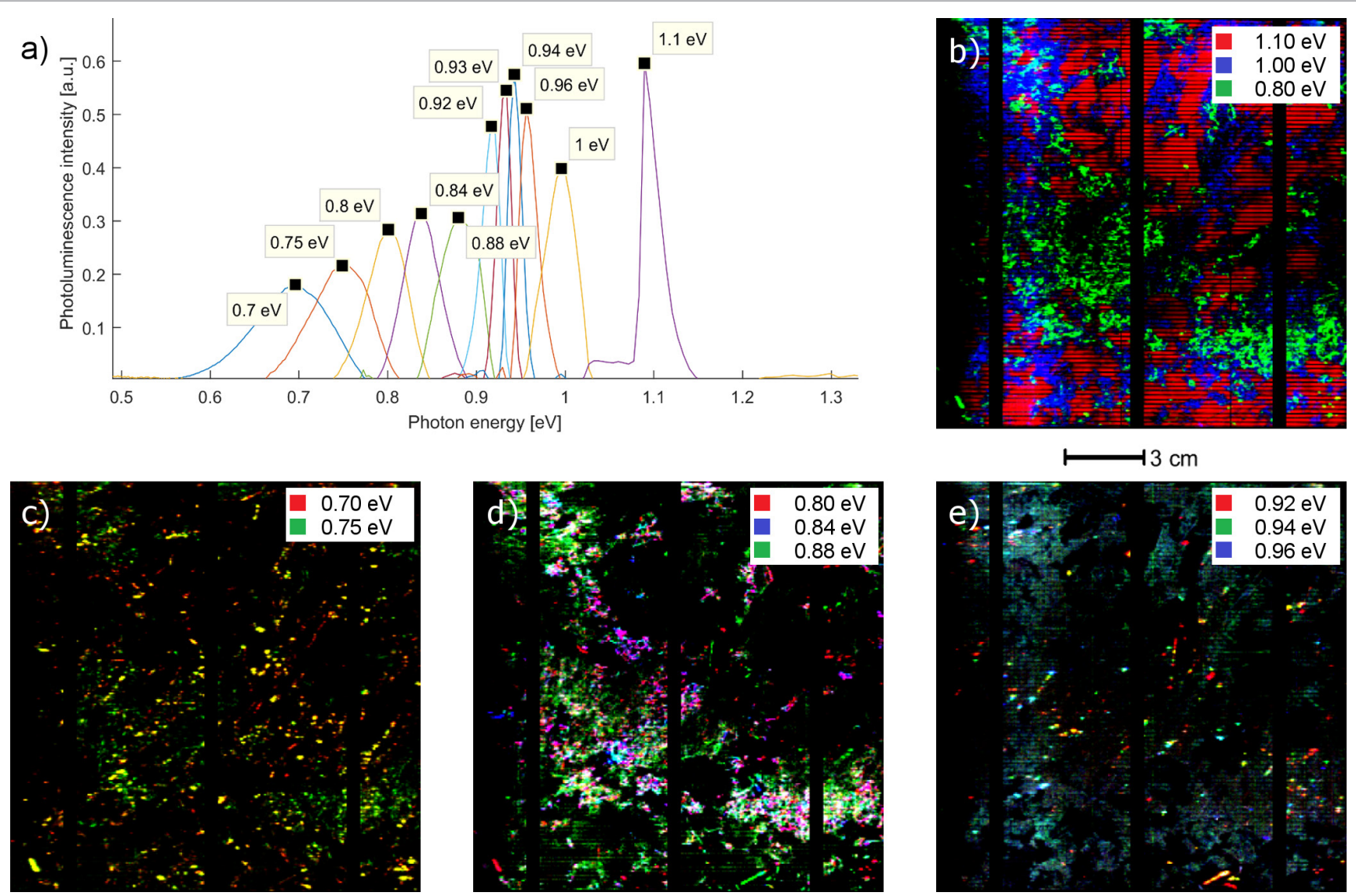

Figure 3. MCR loadings and score images with 11 components of sample 3. Each component has its own colour, but in areas where two or more are present, the colours blend. a) loading plot, b) score images of components identified as BB luminescence, D4 and D1, c) score images with signals at $0.70 \mathrm{eV}$ and $0.75 \mathrm{eV}$, d) score images of $0.80 \mathrm{eV}, 0.84 \mathrm{eV}$ and $0.88 \mathrm{eV}$, e) three of the peaks centred around $0.94 \mathrm{eV}$, often called Very Intense D3 (VID3).
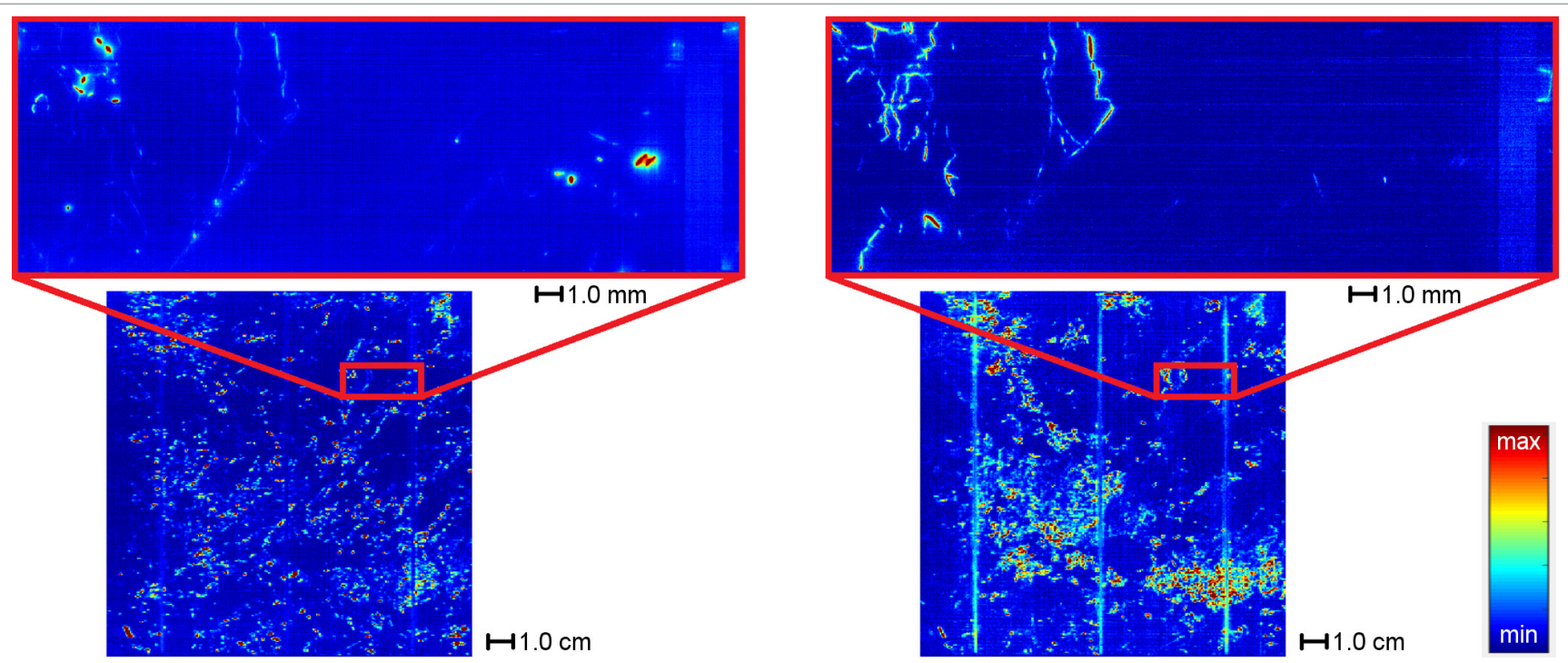

Figure 4. Photoluminescence images across the full solar cell (sample 3) for selected emissions. A small region, indicated by a red rectangle, was imaged with a macro lens setup, yielding a spatial resolution of $30 \mu \mathrm{m}$. These high resolution images show that some of the defect signals originate in grain boundaries $(0.8 \mathrm{eV}$, right panel) whereas other signals show up as singular spots $(0.7 \mathrm{eV}$, left panel). The signal-to-noise with the current setup is unfortunately not high enough to get good MCR analysis with the macro resolution. 


\section{Macro resolution}

Using the macro-lens on the camera and reaching a spatial resolution of $30 \mu \mathrm{m}$ makes it possible to locate and investigate single grain boundaries and the corresponding spectral response as a function of the spectral distribution. This is illustrated in Figure 4 where the $30 \mu \mathrm{m}$ resolution images are shown next to the $500 \mu \mathrm{m}$ ones for two spectral bands at $0.7 \mathrm{eV}$ and $0.8 \mathrm{eV}$, respectively. The defect emission at $0.7 \mathrm{eV}$ can be seen as spots, whereas the emission at $0.8 \mathrm{eV}$ appears along the grain boundaries.

\section{Conclusion}

This work shows that NIR hyperspectral photoluminescence imaging can be used for characterisation of silicon solar cells. Whereas as more traditional methods of characterisation are often long and destructive, hyperspectral imaging allows one to identify and classify defects across the whole sample in a fast and non-destructive way. Moreover, MCR analysis can be used to detect new defect emission lines. Finally, it has been demonstrated how hyperspectral imaging with macro resolution $(30 \mu \mathrm{m})$ reveals information on certain defects. Additional measurements with independent techniques, such as microXRF and dislocation mapping will be carried out extensively to determine the origins of the defects.

\section{References}

1. T. Trupke, R.A. Bardos, M.C. Schubert and W. Warta, "Photoluminescence imaging of silicon wafers",
Appl. Phys. Lett. 89, 044107 (2006). doi: https://doi. org/10.1063/1.2234747

2. T. Trupke, E. Pink, R.A. Bardos and M.D. Abbot, "Spatially resolved series resistance of silicon solar cells obtained from luminescence imaging", Appl. Phys. Lett. 90, 093506 (2007). doi: https://doi.org/10.1063/1.2709630

3. N.A. Drozdov, A.A. Patrin and V.D. Tkachev,

"Recombination radiation on dislocations in silicon," JETP Lett. 23(11), 597 (1976). http://jetpletters.ac.ru/ps/1806/ article 27602.pdf

4. E. Olsen and A.S. Flø, "Spectrally and spatially resolved imaging of photoluminescence in multicrystalline silicon wafers", Appl. Phys. Lett. 99, 011903 (2011). doi: https:// doi.org/10.1063/1.3607307

5. I. Burud, A.S. Flø and E. Olsen, "On the origin of inter band gap radiative emission in crystalline silicon", AlP Advances 2, 042135 (2012). doi: https://doi. org/10.1063/1.4766588

6. R. Tauler, "Multivariate curve resolution applied to second order data”, Chemometr. Intell. Lab. Syst. 30, 133-146 (1995). doi: https://doi.org/10.1016/0169-7439(95)00047-X

7. D. Lausch, K. Petter, B. Henke, R. Bakowskie, S. Schwizer and C. Hagendorf, "Classification of recombination active defect structures in multicrystalline silicon solar cells", Energy Procedia 8, S. 28-34 (2011). doi: https://doi.org/10.1016/j.egypro.2011.06.097

8. D. Lausch, T. Mehl, K. Petter, A. Flø, I. Burud and E. Olsen, "Classification of crystal defects in multicrystalline silicon solar cells and wafer using spectrally and spatially resolved photoluminescence", J. Appl. Phys. 119, 054501 (2016). doi: https://doi.org/10.1063/1.4940711 